\title{
MOLECULAR DOCKING ANALYSIS OF GOSSYPOL ANALOGUES AS HUMAN NEUTROPHIL ELASTASE (HNE), MATRIX METALLOPROTEINASES (MMP 2 AND 9) AND TYROSINASE INHIBITORS
}

\author{
Vishnu Ragavan ${ }^{1}$, Aishwarya Ramesh ${ }^{1}$ and Radhakrishnan \\ Narayanaswamy ${ }^{2, *}$ \\ ${ }^{1}$ Department of Biotechnology, Vel Tech High Tech Dr. Rangarajan Dr. Sakunthala Engineering \\ College, Avadi, Chennai- 600 062, Tamil nadu, India \\ ${ }^{2}$ Department of Biochemistry, St. Peter's Institute of Higher Education and Research (Deemed to \\ be University), Avadi, Chennai-600 054, Tamil nadu, India \\ *E-mail: nrkishnan@gmail.com
}

\begin{abstract}
In the present study, five gossypol analogues such as gossypol, gossypol acetic acid, gossypol polyvinyl pyrrolidine, diamino gossypol and ethyl gossypol were assessed on the docking behaviour of Human neutrophil elastase (HNE), Matrix metalloproteinases (MMP 2 \& 9) and tyrosinase by utilizing PatchDock method. Furthermore, Molecular physicochemical, Drug-likeness, ADME (Absorption, Distribution, Metabolism and Excretion) analyses were also carried out using molinspiration and Swiss ADME methods respectively. The molecular physicochemical investigation showed that five gossypol derivatives showed two to three violations. Similarly, ADME analysis also predicated to have high gastro-intestinal (GI) absorption effect for the five gossypol analogues. The docking studies showed that diamino gossypol exhibited the highest binding energy for all the targeted enzymes such as HNE, MMP 2 and MMP 9 (except tyrosinase). Thus the present investigation provides new knowledge in understanding gossypol analogues as a possible inhibitor against HNE, MMP $2 \& 9$ and tyrosinase.

Keywords: Molecular Docking, Gossypol Analogues, Human Neutrophil Elastase, Matrix Metalloproteinase, Tyrosinase.
\end{abstract}

(C) RASĀYAN. All rights reserved

\section{INTRODUCTION}

Gossypol is a yellowish dye and natural toxin present in the seeds of cotton plants. ${ }^{1}$ It is one of examples of "anti-nutritional" factor occurs in plants. Some of the gossypols derivatives are also known for insecticide activity which is commonly present in the Gossypium species. ${ }^{2}$ Gossypol was first reported by Longmore (1886). ${ }^{3}$ Furthermore, Marchlewski (1899) has purified it, by precipitating using acetic acid which results in formation of gossypol acetic acid. ${ }^{4}$ Gossypol is one of versatile molecule from the genus Gossypium which has been reported to posses various biological activities especially against cancer, HIV, malaria and psoriasis. ${ }^{5}$ Gossypol and its derivatives have been shown to exhibit various pharmaceutical applications like antifertility or contraceptive ${ }^{2,6}$, antioxidant ${ }^{7,8}$, antitumor ${ }^{9-11}$, antiparastic ${ }^{12-14}$, antimicrobial ${ }^{15-17}$ and antiviral agent. ${ }^{18}$

Cosmetic ingredient review (CIR) committee has recommended cottonseed oil derivatives as cosmetic ingredient, however gossypol content in the cosmetic formulations should not exceeds more than 450 ppm level. ${ }^{19}$ Even today cottonseed milk along with coconut milk and jaggery has been consumed by South Indians as traditional health drink. Moreover, Rajam-cottonseed milk mix (Paruthi pal in tamil) is commercial available in the market. The above background prompted us to carry out the present investigation on five selected gossypol analogues which are gossypol, gossypol acetic acid, gossypol polyvinyl pyrrolidine, diamino gossypol and ethyl gossypol. These gossypol analogues were assessed on

Rasayan J. Chem., 13(1), 469-475(2020)

http://dx.doi.org/10.31788/RJC.2020.1315541

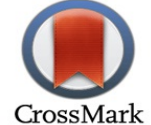


RASĀYAN J. Chem.

Vol. 13 | No. 1 |469 - 475| January - March | 2020

the docking behaviour of Human neutrophil elastase, Matrix metalloproteinases (MMP $2 \&$ 9) and tyrosinase using PatchDock method. Furthermore, Molecular physicochemical, Drug-likeness, ADME (Absorption, Distribution, Metabolism and Excretion) analyses were also carried out in the present study.

\section{EXPERIMENTAL}

\section{Ligand Preparation}

Chemical structures of ligands, i.e., (i) gossypol (ID 3505), (ii) gossypol acetic acid (ID 227456), (iii) gossypol-vinylpyrrolidone (ID 154932), (iv) diaminogossypol (ID 198041) and (v) ethyl gossypol (ID 374353) were downloaded from ChemSpider and PubMed database. The five selected gossypol ligands were prepared according to the reported procedure ${ }^{20-26}$. These prepared structures were used for further Patch Dock.

\section{Target Protein Identification and Preparation}

The three dimensional (3D) structures of the HNE (PDB ID: 1H1B with resolution of $2.00 \mathrm{~A}$ ) and MMP 2 (PDB ID: 1QIB with resolution of 2.80 A), MMP 9 (PDB ID: 4H1Q with resolution of 1.59 A) and Tyrosinase (PDB ID: 2 Y9W with resolution of $2.30 \mathrm{~A}$ ) were obtained from Protein Data Bank (PDB). A chain of these proteins was processed individually by removing another chain (B, C and D) as well as ligands by utilizing UCSF Chimera software. ${ }^{21}$

\section{ADME Analysis}

ADME analysis was performed using Swiss ADME analysis, according to reported procedure. ${ }^{20}$

\section{Docking Studies}

Docking investigations were done by using PatchDock online server. Furthermore, the binding site analysis was done by PyMOL software. ${ }^{23-26}$

\section{RESULTS AND DISCUSSION}

-1 represents the two-dimensional (2D) structures of all five selected gossypol analogues. It is essential to identify the physiochemical and drug-likeness activities of these 5 ligands before performing the docking investigations. Moreover, Lipinski's rule of five (or) thumb rule of five was applied to identify the above said activities and furthermore helps to determine bioavailability of lead molecule $e^{27}$. However, in the present study, gossypol, gossypol-vinylpyrrolidone and diaminogossypol (three ligands) have shown three violations, as shown the Table-2.

Table-1: The Two-dimensional (2D) Structure of Selected Gossypol Analogues

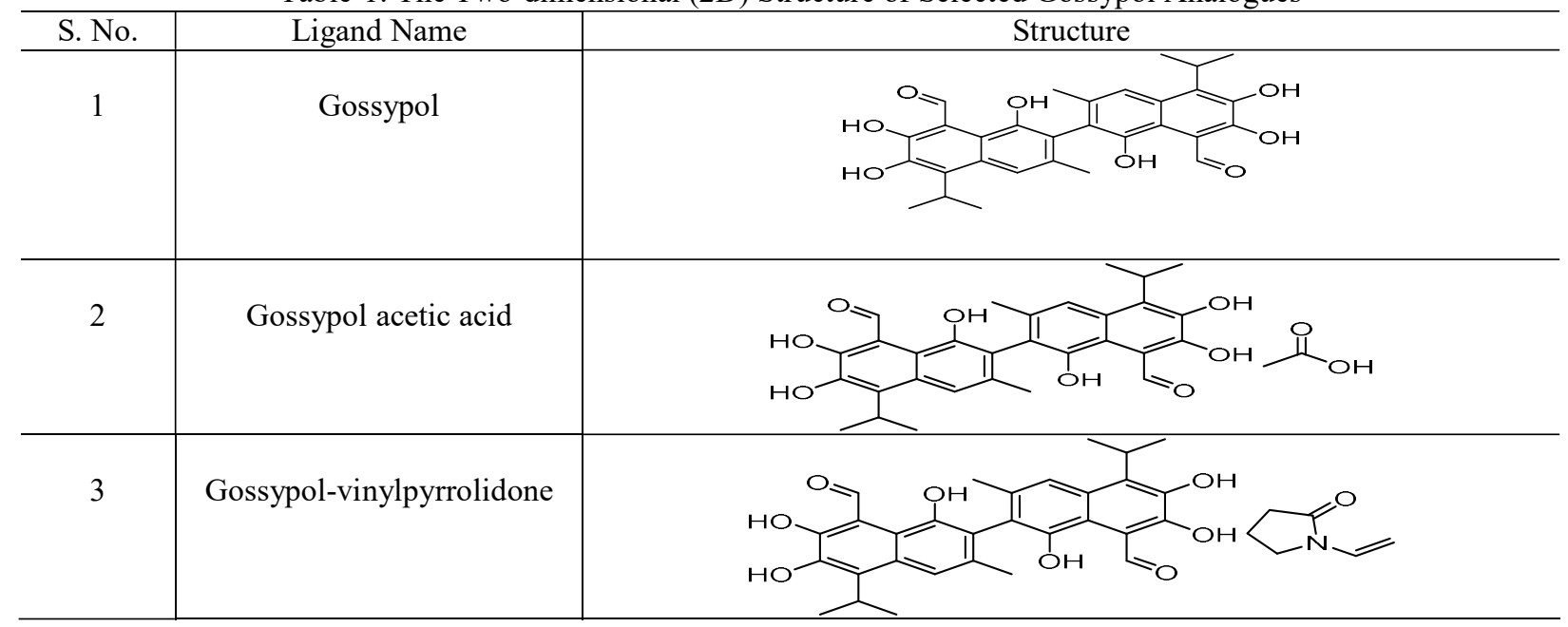


RASĀYAN J. Chem.

Vol. 13 | No. 1 |469 - 475| January - March | 2020

4 Dethyl Gossypol

Table-2: Molecular Physicochemical Analysis of Gossypol Analogues by Utilizing Molinspiration Online Software

\begin{tabular}{c|c|c|c|c|c|c|c|c|c|c}
\hline S. No. & $\begin{array}{c}\text { Gossypol } \\
\text { Analogues }\end{array}$ & $\begin{array}{c}\text { Log } \\
\mathrm{A}^{1}\end{array}$ & $\mathrm{TPSA}^{2}$ & $\begin{array}{c}\text { Natoms } \\
3\end{array}$ & $\mathrm{MW}^{4}$ & $\begin{array}{c}\text { No } \\
\mathrm{N}^{5}\end{array}$ & $\begin{array}{c}\mathrm{nOH}^{6} \\
\mathrm{NH}^{6}\end{array}$ & $\begin{array}{c}\text { Nviolat } \\
\text { ions }^{7}\end{array}$ & $\begin{array}{c}\text { Nrotb } \\
8\end{array}$ & Volume $^{9}$ \\
\hline 1 & Gossypol & 6.57 & 155.51 & 38 & 518.56 & 8 & 6 & 3 & 5 & 462.53 \\
\hline 2 & $\begin{array}{c}\text { Gossypol acetic } \\
\text { acid }\end{array}$ & 7.24 & 135.28 & 38 & 516.59 & 7 & 52 & 2 & 5 & 471.07 \\
\hline 3 & $\begin{array}{c}\text { Gossypol- } \\
\text { vinylpyrrolidone }\end{array}$ & 6.57 & 155.51 & 38 & 518.56 & 8 & 6 & 3 & 5 & 462.53 \\
\hline 4 & Diaminogossypol & 5.39 & 207.56 & 40 & 548.59 & 10 & 10 & 3 & 5 & 485.11 \\
\hline 5 & Ethyl Gossypol & 6.62 & 138.44 & 36 & 490.55 & 7 & 6 & 2 & 4 & 443.55 \\
\hline
\end{tabular}

${ }^{1}$ Octanol-Water partition coefficient, ${ }^{2}$ Polar surface area, ${ }^{3}$ Number of non-hydrogen atoms, ${ }^{4}$ Molecular weight, ${ }^{5}$ Number of hydrogen bond acceptors [ $\mathrm{O}$ and $\mathrm{N}$ atoms], ${ }^{6}$ Number of hydrogen bond donors [ OH and NH groups], ${ }^{7}$

Number of Rule of 5 violations, ${ }^{8}$ Number of rotatable bonds, ${ }^{9}$ Molecular volume.

With regard to Drug-likeness score, if the score is greater than ' 0 ' is active, between -5.0 to -0.0 is moderate active and less than " -5.0 " is inactive. ${ }^{28}$ All the gossypol analogues displayed active bioactivity score towards enzyme inhibitor descriptor as shown in Table-3.

Table-3: Drug-likeness Property Analysis of Gossypol Analogues Using Molinspiration Online Software

\begin{tabular}{c|c|c|c|c|c|c|c}
\hline S.No. & Gossypol analogues & $\begin{array}{c}\text { GPCR } \\
\text { ligand }\end{array}$ & $\begin{array}{c}\text { Ion channel } \\
\text { modulator }\end{array}$ & $\begin{array}{c}\text { Kinase } \\
\text { inhibitor }\end{array}$ & $\begin{array}{c}\text { Nuclear } \\
\text { receptor } \\
\text { ligand }\end{array}$ & $\begin{array}{c}\text { Protease } \\
\text { inhibitor }\end{array}$ & $\begin{array}{c}\text { Enzyme } \\
\text { inhibitor }\end{array}$ \\
\hline 1 & Gossypol & -0.02 & -0.13 & -0.07 & 0.08 & -0.17 & 0.11 \\
\hline 2 & Gossypol acetic acid & -0.02 & -0.14 & -0.12 & 0.11 & -0.15 & 0.13 \\
\hline 3 & $\begin{array}{c}\text { Gossypol- } \\
\text { vinylpyrrolidone }\end{array}$ & -0.02 & -0.13 & -0.07 & 0.08 & -0.17 & 0.11 \\
\hline 4 & Diaminogossypol & -0.06 & -0.19 & -0.16 & -0.14 & -0.23 & 0.09 \\
\hline 5 & Ethyl Gossypol & 0.01 & -0.05 & -0.02 & 0.14 & -0.18 & 0.16 \\
\hline
\end{tabular}

${ }^{1}$ GPCR- G Protein coupled receptors

Similarly, ADME prediction is also essential to perform before the docking investigation and which is recommended in the early stage of Drug discovery, Drug selection and Drug design, due to its unique property. ${ }^{29-30}$ Table-4 displayed the ADME profile of gossypol analogues; all the ligands are predicted to have low gastrointestinal (GI) absorption effect.

Table-4: ADME Analysis of Gossypol Analogues Using Swiss ADME Method

\begin{tabular}{c|c|c|c|c|c|c|c|c|c|c}
\hline S. No. & $\begin{array}{c}\text { Gossypol } \\
\text { Analogues }\end{array}$ & $\mathrm{Gl}^{1}$ & $\begin{array}{c}\mathrm{BB} \\
\mathrm{B}^{2}\end{array}$ & $\begin{array}{c}\mathrm{P}- \\
\mathrm{gp}^{3}\end{array}$ & $\begin{array}{c}\mathrm{CYP} \\
12^{4}\end{array}$ & $\begin{array}{c}\text { CYP219 } \\
5\end{array}$ & $\begin{array}{c}\text { CYP2C9 } \\
6\end{array}$ & $\begin{array}{c}\text { CYP2D6 } \\
7\end{array}$ & $\begin{array}{c}\text { CYP3A } \\
4^{8}\end{array}$ & $\begin{array}{c}\text { Log } \\
\mathrm{Kp}^{9}\end{array}$ \\
\hline 1 & Gossypol & Low & No & No & No & Yes & Yes & No & No & -4.54 \\
\hline 2 & $\begin{array}{c}\text { Gossypol acetic } \\
\text { acid }\end{array}$ & Low & No & No & No & Yes & No & No & No & -4.93 \\
\hline 3 & $\begin{array}{c}\text { Gossypol- } \\
\text { vinylpyrrolidone }\end{array}$ & Low & No & No & No & Yes & No & No & No & -5.03 \\
\hline
\end{tabular}


RASĀYAN J. Chem.

Vol. 13 | No. 1 | 469 - 475| January - March | 2020

\begin{tabular}{c|c|c|c|c|c|c|c|c|c|c}
\hline 4 & $\begin{array}{c}\text { Diaminogossypo } \\
1\end{array}$ & Low & No & No & No & No & Yes & No & No & -5.70 \\
\hline 5 & Ethyl Gossypol & Low & No & No & No & Yes & Yes & No & No & -4.39 \\
\hline
\end{tabular}

Gossypol has been reported to inhibit lactate dehydrogenase (LDH) activity ${ }^{31}$ and similarly it has been reported to inhibit a number of oxidoreductases activities. ${ }^{32}$ Gossypol has been reported to inhibit both arachidonate 5 and 12 Lipoxygenases $\left(5,12\right.$ - LOX) activities. ${ }^{33}$ In the present study human nucleophil elastase (HNE) is one of the target protein/enzyme, which its docking investigation showed diaminogossypol has the highest atomic contant energy $(-373.10 \mathrm{kcal} / \mathrm{mol})$. Interestingly, diaminogossypol (Fig.-1) and ethyl gossypol have shown interaction with Asn 61 amino acid residue of HNE as given in the Table- 5 .

Table-5: Binding Energy Analysis of Gossypol Analogues with Human Neutrophil Elastase (HNE) using PatchDock

\begin{tabular}{|c|c|c|c|c|}
\hline \multicolumn{5}{|c|}{ Method } \\
\hline S. No. & Gossypol Analogues & $-\mathrm{ACE}^{* *}(-\mathrm{kcal} / \mathrm{mol})$ & $\begin{array}{c}\text { Interaction of Amino } \\
\text { Acid Residue }\end{array}$ & Bond Distance $(\AA)$ \\
\hline 1 & Gossypol & 277.10 & Val 216 & 2.9 \\
\hline 2 & Gossypol acetic acid & 79.47 & $\begin{array}{l}\text { Asn } 101 \\
\text { Arg } 129\end{array}$ & $\begin{array}{l}3.1 \\
3.0\end{array}$ \\
\hline 3 & Gossypol-vinylpyrrolidone & 260.50 & $\begin{array}{l}\text { Glu } 156 \\
\text { Arg } 147\end{array}$ & $\begin{array}{l}2.5 \\
3.5\end{array}$ \\
\hline 4 & Diaminogossypol & 373.10 & Asn 61 & 3.1 \\
\hline 5 & Ethyl Gossypol & 288.40 & Asn 61 & 3.1 \\
\hline
\end{tabular}

Atomic contact energy

Above outcome was in good agreement with previous publication, where gossypol (from Thespesia populnea) shown to inhibit elastase activity. ${ }^{34}$

MMPs are a group of metallo-enzymes (especially contains zinc metal) that to particularly MMP 2 and MMP 9 have been elevated in the pathological disesases like ageing, cancer, inflammation and wound healing. ${ }^{21}$ In the present study, MMP 2 and MMP 9 are another two target proteins/enzymes where the docking investigation showed diaminogossypol has the highest atomic contact energy with of MMP 2 ($568.66 \mathrm{kcal} / \mathrm{mol})$ and MMP $9(-583.33 \mathrm{kcal} / \mathrm{mol})$ respectively. Interestingly, gossypol, gossypol acetic acid and diaminogossypol have shown interaction with Thr 227 amino acid residue of MMP 2 as tabilated in Table-6.

Table-6: Binding Energy Analysis of Gossypol Analogues with Matrix Metalloproteinase (MMP 2) Using PatchDock Method

\begin{tabular}{c|c|c|c|c}
\hline S. No. & Gossypol Analogues & $-\mathrm{ACE}^{* *}(-\mathrm{kcal} / \mathrm{mol})$ & $\begin{array}{c}\text { Interaction of Amino Acid } \\
\text { Residue }\end{array}$ & $\begin{array}{c}\text { Bond Distance } \\
(\AA)\end{array}$ \\
\hline 1 & Gossypol & 526.81 & Gly 216 and Thr 227 & $2.6 \& 2.9$ \\
\hline 2 & Gossypol acetic acid & 549.08 & Thr 227 & 3.4 \\
\hline 3 & Gossypol- & 556.92 & Ala 165 & 2.3 \\
& vinylpyrrolidone & & Leu 164 & 2.9 \\
& & & Ala 165 & 3.3 \\
\hline 4 & Diaminogossypol & 568.66 & Pro 215 & 1.6 \\
& & & Tyr 223 & 2.1 \\
& & & Gly 216 & 2.1 \\
& & & Gly 162 & 3.1 \\
\hline 5 & Ethyl Gossypol & & Thr 227 & $1.8 \& 2.7$ \\
\hline
\end{tabular}

Atomic contact energy 
RASĀYAN J. Chem.

Vol. 13 | No. 1 |469 - 475| January - March | 2020

Similarly gossypol, gossypol acetic acid, gossypol polyvinyl pyrrolidine and diamino gossypol showed interaction with Arg 249 amino acid residue of MMP 9 as given in Table-7. The present result was in good align with previous literature, where gossypol (from Abelmoscus esculentus) has shown to dock with MMP 9 using molecular docking method. ${ }^{35}$

Table-7: Binding Energy Analysis of Gossypol Analogues with Matrix Metalloproteinase (MMP-9) Using PatchDock Method

\begin{tabular}{c|c|c|c|c}
\hline S. No. & $\begin{array}{c}\text { Gossypol } \\
\text { Analogues }\end{array}$ & -ACE** $(-\mathrm{kcal} / \mathrm{mol})$ & $\begin{array}{c}\text { Interaction of Amino Acid } \\
\text { Residue }\end{array}$ & $\begin{array}{c}\text { Bond Distance } \\
(\AA)\end{array}$ \\
\hline 1 & Gossypol & 530.55 & Leu 188 & 3.4 \\
& & & Leu 188 & 3.5 \\
& & & Arg 249 & 2.5 \\
\hline 2 & Gossypol acetic & 553.43 & Arg 249 & 3.2 \\
& acid & & Pro 240 & 3.5 \\
\hline 3 & Gossypol- & 568.84 & Arg 249 & 3.3 \\
& vinylpyrrolidone & & & 2.8 \\
& Diaminogossypol & 583.33 & Arg 249 & 3.5 \\
& & & Pro 240 & 3.3 \\
& & & Leu 188 & 3.4 \\
\hline \multirow{2}{*}{5} & Ethyl Gossypol & & Ala 189 & Ala 189 \\
& & 552.38 & Pro 240 & 3.1 \\
& & & Glu 241 & 2.8 \\
& & & Leu 188 & 3.3 \\
\hline
\end{tabular}

\footnotetext{
${ }^{* *}$ Atomic contact energy
}

Tyrosinase is the main regulatory enzyme in melanogenesis pathway especially in the initially two stages such as (i) tyrosine hydroxylation to 3, 4-dihydroxyphenylalanine (DOPA) and (ii) the oxidation of DOPA to dopoquinone ${ }^{36}$. In this present investigation tyrosinase is the another target enzyme where the docking result showed gossypol-vinylpyrrolidone has the highest atomic contact energy (-137.04 $\mathrm{kcal} / \mathrm{mol})$. Similarly, gossypol acetic acid showed very atomic contact energy $(+19.22 \mathrm{kcal} / \mathrm{mol})$ with tyrosinase, which might due to unfavorable interactions phenomenon as reported by Castro and coworkers. ${ }^{37}$ Interestingly, gossypol and gossypol-vinylpyrrolidone have shown interaction with Gln74 amino acid residue of tyrosinase as tabulated in Table- 8 .

Table-8: Binding Energy Analysis of Gossypol Analogues with Tyrosinase Using PatchDock Method

\begin{tabular}{c|c|c|c|c}
\hline S. No. & Gossypol Analogues & -ACE** $(-\mathrm{kcal} / \mathrm{mol})$ & $\begin{array}{c}\text { Interaction of Amino } \\
\text { Acid Residue }\end{array}$ & $\begin{array}{c}\text { Bond } \\
\text { Distance }(\AA)\end{array}$ \\
\hline 1 & Gossypol & 44.94 & Glu67 & 2.3 \\
& & & Lys70 & 3.2 \\
& & & Gln74 & 2.3 \\
& & & Gln74 & 3.3 \\
\hline 2 & Gossypol acetic acid & +19.22 & Thr308 & 3.2 \\
& & & Lys372 & 2.4 \\
\hline 3 & Gossypol-vinylpyrrolidone & 137.04 & Gln73 & 2.6 \\
\hline 4 & & & Gln74 & 3.2 \\
\hline & Diaminogossypol & 77.24 & Gln72 & 3.1 \\
& & & Tyr343 & 2.2 \\
\hline 5 & Ethyl Gossypol & & Tyr343 & 2.8 \\
\hline
\end{tabular}

Atomic contact energy 
RASĀYAN J. Chem.

Vol. 13 | No. 1 |469 - 475| January - March | 2020
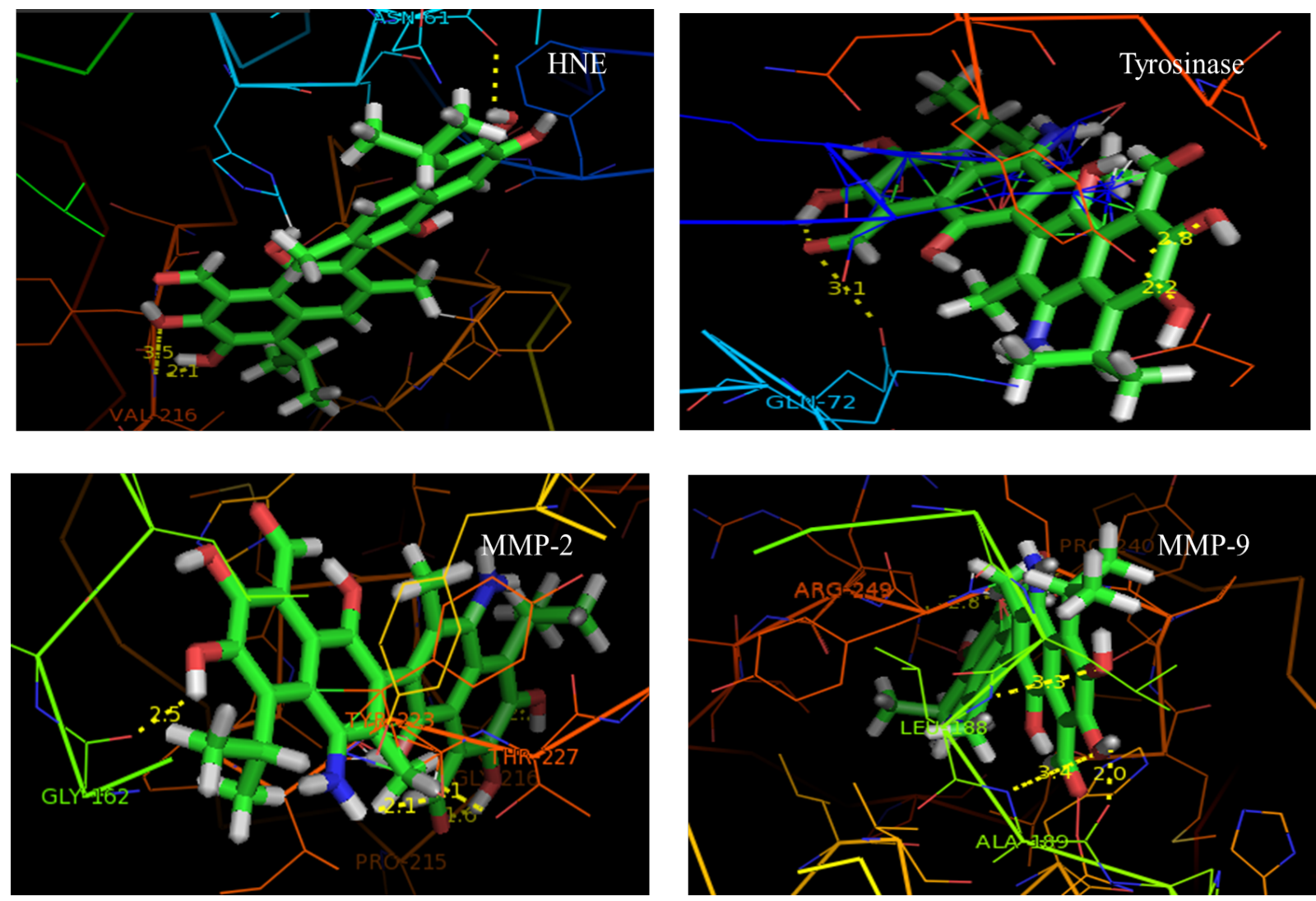

Fig.-1: Represents the Diaminogossypol has shown to interaction with all four target enzymes, especially with the Amino Acid Residues (Top row- Left (Human neutrophil elastase-HNE) and Right (Tyrosinase); Bottom row: Left (Matrix metalloproteinase 2) and Right (Matrix metalloproteinase 9).

\section{CONCLUSION}

In conclusion, gossypol derivatives have shown potential to dock with all the four selected proteins (or) enzymes (namely HNE, MMP 2 \& MMP 9 and tyrosinase). The present docking investigation has shown diamino gossypol as exhibited the maximum atomic contact energy for three selected enzymes, except for tyrosinase. Hence, the present study strongly suggested that potential of these five gossypol derivatives as HNE, MMP 2 \& MMP 9 and tyrosinase inhibitory agents.

\section{ACKNOWLEDGEMENT}

The authors wish to thank Dr. V. Vijayakumar, Research Associate, Department of Chemistry, Anna University, Chennai for his help to complete this work.

\section{REFERENCES}

1. H.G. Jung, G.C. Fahey, Journal of Animal Science, 57, 206(1983), DOI:10.2527/jas1983.571206x

2. X. Wang, C.P. Howell, F. Chen, J. Yin, Advances in Food and Nutrition Research, 58, 215(2009), DOI: 10.1016/S1043-4526(09)58006-0

3. J. Longmore, Indian Journal of Chemistry, 5, 200(1886).

4. L. Marchlewski, Journal Fur Praktische Chemie, 60, 84(1899), DOI:10.1002/prac.18990600108

5. H. Keshmiri-Neghab, B. Goliaei, Pharmaceutical Biology, 52, 124(2014), DOI: $10.3109 / 13880209.2013 .832776$

6. E.M. Coutinho. Contraception, 65, 259(2002), DOI:10.1016/S0010-7824(02)00294-9

7. M. Ligueros, D. Jeoung, B. Tang, D. Hochhauser, M.M. Reidenberg, M. Sonenberg, British Journal of Cancer, 76, 21(1997), DOI:10.1038/bjc.1997.330

8. E.L. Hove, Journal of Biological Chemistry, 156, 633(1944).

9. S. Kitada, M. Leone, S. Sareth, D. Zhai, J.C. Reed, M. Pellecchia, Journal of Medicinal Chemistry, 46, 4259(2003), DOI:10.1021/jm030190z 
10. S. Liu, S.K, Kulp, Y. Sugimoto, J. Jiang, H.L. Chang, M.K. Dowd, P. Wan, Y.C. Lin, Anticancer Research, 22, 33(2002).

11. D. Zhai, C. Jin, A.C. Satterthwaite, J.C, Reed, Cell Death and Differentiation, 13, 1419(2006), DOI: 10.1038/sj.cdd. 4401937

12. K. Mendis, B.J. Sina, P. Marchesini, R. Carter, American Journal Tropical Medicine and Hygiene, 64, 97(2001), DOI:10.4269/ajtmh.2001.64.97

13. V. Razakantonina, P.P. Nguyen Kim, G. Jaureguiberry, Parasitology Research, 86, 665(2000).

14. R.E. Royer, L.M. Deck, N.M. Campos, L.A. Hunsaker, D.L. Vander, Journal of Medicinal Chemistry, 29, 1799(1986), DOI:10.1021/jm00159a043

15. A.A. Bell, R.D. Stipanovic, Mycopathologia, 65, 91(1978), DOI:10.1007/BF00447180

16. P. Margalith, Applied and Environmental Microbiology, 15, 952(1967).

17. D.V. Vandehra, N.R Kalla, M. Sexena, R. Hashia, P. Kaur, I.K. Gupta, IRCS Medical Science (Biochemistry), 13, 10(1985).

18. T.S. Lin, R.F. Schinazi, B.P. Griffith, E.M, August, B.F. Eriksson, D.K. Zheng, L.A. Huang, W.H. Prusoff, Antimicrobial Agents and Chemotherapy, 33, 2149(1993), DOI:10.1128/AAC.33.12.2149

19. M. Bindu Nair, International Journal of Toxicology, 20, 21(2001), DOI: 10.1080/1091581017503009371

20. V. Vijayakumar, N. Radhakrishnan, C. Rameshkumar, IEEE International Conference on Smart Technologies and Management for Computing, Communication, Controls, Energy and Materials, 513(2017), DOI:10.1109/ICSTM.2017.8089213

21. N. Radhakrishnan, K.W. Lam, I.S. Intan, International Journal of Food Properties, 18, 2155(2015). DOI: $10.1080 / 10942912.2014 .963870$

22. M. Muchtaridi, S. Megantara, D. Dermawan, M. Yusuf, Rasayan Journal of Chemistry, 12, 1927(2019), DOI: 10.31788/RJC.2019.1245391

23. B. Geetha, G. Swarnalatha, G.V. Subba Reddy, Rasayan Journal of Chemistry, 12, 1063(2019), DOI: 10.31788/RJC.2019.1235165a

24. M. Malathi, D. Prabhakara Chary, Rasayan Journal of Chemistry, 12, 1347(2019), DOI: 10.31788/RJC.2019.1235290

25. I. Irfan, M. Irfan, M. Abid, A. Azam, Rasayan Journal of Chemistry, 12, 2082(2019), DOI: 10.31788/RJC.2019.1245320

26. V. Vijayakumar, A. Prabakaran, N. Radhakrishnan, C. Rameshkumar, S. Muthu, E.I. Paulraj, Journal of Molecular Structure, 1179, 325(2018), DOI:10.1016/j.molstruc.2018.11.018

27. C.A. Lipinski, F. Lambardo, B.W. Dominy, P.J. Feeney, Advanced Drug Delivery Reviews, 23, 3(1997), DOI: 10.1016/S0169-409X(96)00423-1

28. S. Singh, A.K. Gupta, A. Verma. Research Journal of Pharmaceutical, Biological and Chemical Sciences, 4, 876(2013).

29. V. Vijayakumar, N. Radhakrishnan, C. Rameshkumar, Asian Journal of Pharmaceutical and Clinical Research, 10, 383(2017), DOI:10.22159/ajpcr.2017.v10i11.20999

30. J. Wang, L. Urban, Drug Discovery World, 5, 73(2004).

31. C.Y. Lee, H.V. Malling. Federation Proceedings, 40, 718(1981).

32. N.M. Gerez deBurgos, C. Burgos, E.E. Montamat, L.E. Rovai, A. Blanco, Biochemical Pharmacology, 33, 955(1984), DOI:10.1016/0006-2952(84)90500-8

33. Y. Hamasaki, H.H. Tai, Biochimica et Biophysica Acta, 834, 37(1985), DOI:10.1016/00052760(85) $90173-0$

34. T. Annamalai, G. Venkateswara Rao, T. Mukhopadhyay, Der Pharmacia Lettre, 5, 312(2013).

35. M.S. Kumaran, P. Sivaselvi, P. Brindha, T. Vimala, World Journal Pharmaceutical Sciences, 2, 253(2004).

36. N. Radhakrishnan, S. Ashok, V. Kavitha, G. Rameshkumar, A. Gnanamani, Journal of Chemical and Pharmaceutical Research, 5, 320(2013).

37. J.S. Castro, B. Trzaskowski, P.A. Deymier, J. Bucay, L. Adamowicz, J.B. Hoying, Materials Science and Engineering: C, 29, 1609(2009), DOI:10.1016/j.msec.2008.12.026

[RJC-5541/2019] 